\title{
Influence of lattice heating time on femtosecond laser-induced strain waves in InSb
}

\author{
F. S. Krasniqi, * S. L. Johnson, P. Beaud, M. Kaiser, D. Grolimund, and G. Ingold \\ Swiss Light Source, Paul Scherrer Institute, CH-5232 Villigen PSI, Switzerland
}

(Received 29 May 2008; published 6 November 2008)

\begin{abstract}
Femtosecond laser-induced strain waves in InSb are studied by means of time-resolved x-ray diffraction. The temporal evolution of the measured x-ray diffracted intensity reveals that the lattice dynamics depends on the time scale of energy transfer from excited carriers to the lattice. A framework that accounts for this energytransfer time (lattice heating time) is presented and applied to model the fluence dependence of the transient $\mathrm{x}$-ray diffraction data. In this model the initial strain wave dynamics depends crucially on the lattice heating time, which decreases with increasing fluence.
\end{abstract}

DOI: 10.1103/PhysRevB.78.174302

PACS number(s): 62.30.+d, 63.20.-e

\section{INTRODUCTION}

Above band-gap excitation of a semiconductor crystal with a subpicosecond laser pulse with pulse energies just below the damage threshold puts the crystal into a highly stressed state. Stress is typically relieved by lattice expansion that starts at the crystal surface and subsequently drives a traveling expansion and compression strain wave into the bulk at the longitudinal speed of sound.,2 The timedependent strain wave can be thought as a superposition of coherent phonons with wave vectors centered approximately about the inverse of laser penetration depth. ${ }^{3}$ These transient coherent lattice dynamics have been studied in a variety of materials both by observing the resultant changes in optical properties (reflectivity) $1,4,5$ and by using $\mathrm{x}$-ray diffraction. ${ }^{2,6-8}$ In contrast to optical reflectivity measurements, time-resolved $\mathrm{x}$-ray diffraction can directly observe the small shifts in interatomic distance associated with a strain wave as it propagates into the bulk of the crystal. The sensitivity of x-ray diffraction to coherent lattice dynamics has been demonstrated in many experiments in both Bragg and Laue geometries. ${ }^{9}$ Generally, these studies are useful in deducing the acoustic properties of the excited crystal and testing models of electron-phonon coupling. $3,4,7$

A simple model that describes the generation of a strain wave in a laser-excited solid was proposed by Thomsen et $a l .{ }^{1}$ They solved the elastic equations assuming that thermal stress is instantaneously generated in an absorbing solid. This model has successfully been applied to explain the results of both optical scattering and $\mathrm{x}$-ray diffraction measurements. ${ }^{1,2,9}$ In principle, one might expect that the model predicts reasonably the structure of the strain wave at times well after carrier-lattice thermalization. During the thermalization process, on the other hand, the assumption of instantaneous heating may overestimate the strain. For instance, in polar semiconductors such as InSb, carrier-lattice energy exchange is thought to be mediated by longitudinaloptical (LO) phonons. ${ }^{10}$ In these systems, the excess energy of photoexcited carriers is first transferred to small momentum LO phonons, which subsequently decay to acoustic phonons due to the anharmonicity of the crystal potential. The intrinsic lifetime of LO phonons is expected to govern the carrier-lattice thermalization dynamics ${ }^{10,11}$ and thus the thermal component of the strain evolution. Intervalley scat- tering of carriers, significant for dense electron-hole plasmas and high carrier energies, may further influence the thermalization time scale by reheating the carriers and reducing the cooling rate, whereas deformation-potential and piezoelectric scatterings with acoustic phonons are important for carrier excess energies smaller than the LO phonon energy. ${ }^{10,12}$

In the present paper we have employed time-resolved $\mathrm{x}$-ray diffraction using the femtosecond "slicing" source at the Swiss Light Source (SLS) (Ref. 13) to investigate the fluence dependence of the lattice heating time in laserexcited InSb. A model for the laser-excited strain waves is developed assuming that energy transfer from photoexcited carriers to the lattice is mediated mainly by LO phonons. We give an analytical expression for the laser-induced strain wave that takes into account the energy-transfer time from the excited electrons to the lattice. This model is an extension of the Thomsen model; it produces Thomsen-type strain profiles in the limit of instantaneous heating (i.e., when the lattice heating time tends to zero). The x-ray diffraction from a laser strained crystal is calculated using the Takagi-Taupin dynamical theory for the depth-dependent strain gradients. ${ }^{14}$ We find that the lattice heating time does affect the time evolution of the x-ray diffracted intensity during carrierlattice thermalization.

The paper is organized as follows. In Sec. II we briefly describe the experimental setup. Based on results from previous work on lattice heating and strain wave generation, Sec. III discusses the effect of laser heating on the strain wave. Here we present a model that can be used to calculate strain wave profiles, including the lattice heating time. In Sec. IV we calculate the x-ray diffraction from a strained crystal. In Sec. V we present and discuss our experimental results in the framework of models presented in two previous sections. Finally, in Sec. VI we present the conclusions of our work.

\section{EXPERIMENTAL SETUP}

The experiment was performed at the SLS using a tunable femtosecond undulator hard x-ray source. Short $140 \pm 30$ fs $\mathrm{X}$-ray pulses are generated using the electron-beam slicing technique $^{13,15}$ at a repetition rate of $1 \mathrm{kHz}$. Two mirrors focus the x-ray beam onto the sample. The first is a horizontally mounted grazing incidence toroidal mirror placed $15 \mathrm{~m}$ be- 


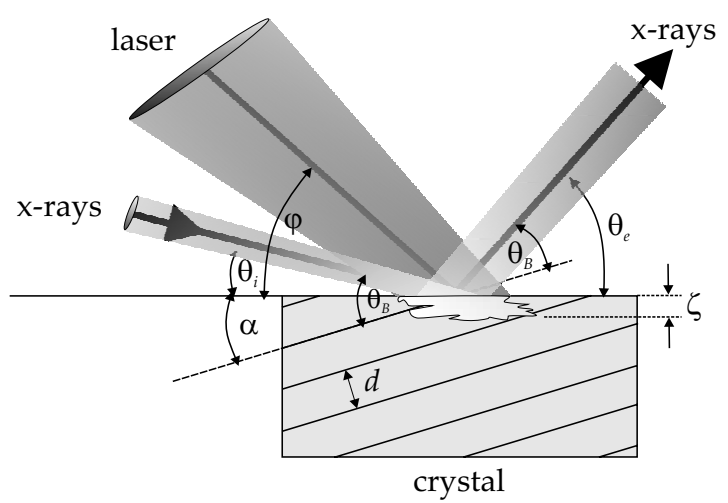

FIG. 1. Scheme of the asymmetric Bragg geometry. The grazing angle of incidence of $\mathrm{x}$ rays with respect to the crystal surface is denoted by $\theta_{i} ; \alpha$ is the asymmetry angle, $\theta_{B}$ is the Bragg angle, $\theta_{e}$ is the exit angle, $\varphi$ is the angle between the laser beam and the crystal surface, and $\zeta$ is the laser penetration depth.

fore the sample that both collimates the beam vertically and brings the $\mathrm{x}$ rays to a horizontal focus of $250 \mu \mathrm{m}$ at the sample. The second mirror is an elliptically bent grazing incidence optic positioned $43 \mathrm{~cm}$ before the sample. This mirror focuses the beam vertically to less than $10 \mu \mathrm{m}$. Between this last mirror and the sample, a double multilayer (Mo/ $\mathrm{B}_{4} \mathrm{C}, 25 \AA$ period) monochromator selects an $\mathrm{x}$-ray energy of $5.9 \mathrm{keV}$ with a bandwidth of $1.2 \%$. At a variable time relative to the arrival of the $\mathrm{x}$-ray pulses, a $p$-polarized femtosecond laser pump pulse $[\lambda=800 \mathrm{~nm}$, full width at half maximum $(\mathrm{FWHM})=120 \mathrm{fs}]$ excites the sample with a $16^{\circ}$ incidence angle, as measured from the crystal surface.

The x-ray diffraction measurements were performed on an asymmetrically cut InSb single crystal (500 $\mu \mathrm{m}$ thick) as drawn in Fig. 1. The surface of the crystal was cut to an angle $\alpha=15.5^{\circ}$ from the (111) lattice planes. At $5.9 \mathrm{keV}$ $\mathrm{x}$-ray energy the (111) Bragg angle is $16.3^{\circ}$. The grazing angle of incidence of $\mathrm{x}$-rays with respect to the surface was $\theta_{i}=\theta_{B}-\alpha=0.8^{\circ}$, and the angle of the exiting X-ray beam with respect to the crystal surface was $\theta_{e}=\theta_{B}+\alpha=31.8^{\circ}$. The signal was measured by an avalanche photodiode detector.

The asymmetric Bragg geometry provides a better match between the penetration depths of laser and $\mathrm{x}$ rays than does a symmetric diffraction geometry. At grazing incidence angles below $1^{\circ}$ at these energies in InSb, photoabsorption of $\mathrm{x}$ rays limits the penetration into the crystal. Under this approximation, the x-ray penetration depth can be written as

$$
\zeta_{x}=\frac{1}{\mu}\left[\frac{\sin \left(\theta_{B}-\alpha\right)}{1+|b|}\right],
$$

where $\mu$ is the linear absorption coefficient and $b$ is the asymmetry parameter defined as $b=-\sin \left(\theta_{B}-\alpha\right) / \sin \left(\theta_{B}+\alpha\right)$. For $x$ rays at $5.9 \mathrm{keV}, \mu \approx 3.144 \times 10^{5} \mathrm{~m}^{-1}$ (Ref. 16) and Eq. (1) gives a penetration depth $\zeta_{x} \approx 43 \mathrm{~nm}$.

The laser spot size on the sample was approximately seven times larger than x-ray spot size to ensure probing of a homogeneously excited area. The loss in temporal resolution due to the size of the $\mathrm{x}$-ray beam and the angle between the pump and x-ray beams was less than 90 fs.

\section{GENERATION OF STRAIN WAVES}

The fundamental interactions which occur during and following the absorption of subpicosecond, above-band-gapenergy laser pulses have been described by many authors. ${ }^{17}$ In this section we give a brief summary of the processes that are relevant to our work and the necessary considerations to incorporate the effects of lattice heating. The main objective of this section is to develop an expression for the lattice temperature that will be presented in Sec. III A which we will use in Sec. III B to model strain waves and subsequently in the analysis and discussion of the experimental results.

\section{A. Lattice heating}

When a semiconductor crystal is excited with visible or near-infrared photons of energy $\hbar \omega$ larger than the energy gap $E_{g}$, electrons are excited out of valence-band (VB) states into conduction-band (CB) states. Neglecting recombination and diffusion during excitation, the evolution of the carrier density profile during and immediately after the laser pulse can be described by the following partial differential equations for the carrier density $N_{e}(z, t)$ and the pump intensity $I(z, t)$ (Ref. 18):

$$
\frac{\partial}{\partial z} I(z, t)=-\left[\alpha_{0}+\alpha_{\mathrm{fc}}(z, t)+\beta_{\mathrm{TPA}} I(z, t)\right] I(z, t)
$$

and

$$
\frac{\partial}{\partial t} N_{e}(z, t)=\left[\alpha_{0}+\frac{1}{2} \beta_{\mathrm{TPA}} I(z, t)\right] \frac{I(z, t)}{\hbar \omega} .
$$

Here, $z$ denotes the spatial coordinate perpendicular to the surface, $\alpha_{0}$ and $\beta_{\mathrm{TPA}}$ account for linear and two-photon absorption (for $\mathrm{InSb}$ at $\lambda_{\text {las }}=800 \mathrm{~nm}, \alpha_{0} \approx 1.087 \times 10^{7} \mathrm{~m}^{-1}$ and $\left.\beta_{\mathrm{TPA}} \approx 8 \times 10^{-5} \mathrm{~m} / \mathrm{GW}\right),{ }^{19,20}$ and intraband free-carrier absorption is calculated using the Drude model for the dielectric constant ${ }^{21} \varepsilon\left(N_{e}\right), \alpha_{\mathrm{fc}} \sim \varepsilon_{2} /\left[2 \sqrt{\varepsilon_{1}+\sqrt{\left(\varepsilon_{1}^{2}+\varepsilon_{2}^{2}\right)}}\right]$, where $\varepsilon_{1}\left(N_{e}\right)$ and $\varepsilon_{2}\left(N_{e}\right)$ are the real and imaginary parts of $\varepsilon\left(N_{e}\right)$, respectively. These equations can be solved using boundary conditions $I(z=0, t)=I_{0} e^{-t^{2} / \tau_{0}^{2}}$ and $N(z, t=0)=N_{0}$, where $N_{0}$ $\approx 2 \times 10^{16} \mathrm{~cm}^{-3}$ is the equilibrium carrier concentration at $T=300 \mathrm{~K}$.

Following Ref. 22 the evolution of the carrier density at later times can be described by the differential equation

$$
\frac{\partial N_{e}}{\partial t}=D_{a} \frac{\partial^{2} N_{e}}{\partial z^{2}}-C_{a} N_{e}^{2}
$$

Here, the first term on the right-hand side describes the carrier diffusion characterized by the ambipolar diffusion coefficient $D_{a}$ that depends in general on the carrier density and temperature. ${ }^{23,24}$ For InSb under high carrier density (on the order of $10^{20} \mathrm{~cm}^{-3}$ ) and excess energy (about $1 \mathrm{eV}$ ) conditions $D_{a} \approx 40 \mathrm{~cm}^{2} / \mathrm{s}$ has been deduced from optical reflectivity measurements. ${ }^{22}$ For carrier densities on the order of $10^{21} \mathrm{~cm}^{-3}$ band-gap renormalization ${ }^{23,24}$ slows down the diffusion; in the case where this effect is ignored, the carrier density in the excitation depth is not more than $20 \%$ larger because of the large Auger recombination rate at these den- 
sities. Auger recombination characterized by the recombination coefficient $C_{a}$ is described by the second term. In highly excited InSb, due to the screening of the Coulomb potential that mediates the carrier-carrier interaction, an Auger recombination rate with quadratic dependence on $N_{e}$ is shown to be more successful, with $C_{a} \approx 1.5 \times 10^{-9} \mathrm{~cm}^{3} / \mathrm{s}^{22}$ Other recombination mechanisms, such as radiative recombination, are negligible for time scales $<1$ ns (Ref. 11). This equation does not distinguish between the $\Gamma$ and $L$ valleys in the CB. However, by using the model described in Ref. 25 (including diffusion in the $\Gamma$ valley with ambipolar diffusion coefficient $D_{a}$ and recombination in both $\Gamma$ and $L$ valleys with the same Auger recombination coefficient $C_{a}$ ) and assuming an intervalley scattering time $\Gamma \rightarrow L$ comparable to the laser-pulse duration, $L \rightarrow \Gamma$ scattering time of $1 \mathrm{ps}$, and optical phonon emission time of $150 \mathrm{fs}$, the time evolution of the carrier density differs not much from that computed using Eq. (4), with discrepancies smaller than $30 \%$ at times $<5$ ps, whereas at later times the discrepancy is larger but the values of carrier density are of the same order magnitude. Variation of the above-mentioned scattering times by $\sim 40 \%$ increases the discrepancy by $\sim 14 \%$.

A monochromatic laser pulse excites electron-hole pairs at specific points in the band structure determined by the condition $E_{c}(\mathbf{k})-E_{v}(\mathbf{k})=\hbar \omega$, where $E_{c}, E_{v}$, and $\hbar \omega$ are the $\mathrm{CB}, \mathrm{VB}$, and laser photon energies, respectively. The big difference in the curvatures of the $\mathrm{CB}$ and VB (Ref. 27) at $\hbar \omega \approx 1.55 \mathrm{eV}$ implies that most of the excess energies $\Delta E_{c}$ ( $c=e, h$ for electrons and holes, respectively) reside initially in the electrons rather than in the holes. ${ }^{50}$ Although the excited carriers initially have a nonthermal distribution, scattering processes such as carrier-carrier (and to lesser extend carrier-phonon) scattering thermalize electrons and holes in a very short time scale on the order of $100 \mathrm{fs} .^{11,28}$ Under the assumption that most of the photon energies are translated into the kinetic energy of the electrons, we may estimate the electron temperature $T_{e 0}$ as

$$
T_{e 0} \approx \frac{2 \Delta E_{e}}{3 k_{B}},
$$

where $k_{B}$ is the Boltzmann constant.

It is generally recognized that in semiconductors of polar character, carriers lose their excess energy primarily by emitting LO phonons via Fröhlich interaction. ${ }^{11,29,30}$ This interaction favors optical phonons near the center of the Brillouin zone (BZ) since the rate matrix elements are proportional to $1 / q^{2}$, where $q$ is the phonon wave vector. ${ }^{31}$ If excitation of carriers takes place in the $\Gamma$ valley, the photoexcited carrier density is high $\left(\gtrsim 10^{20} \mathrm{~cm}^{-3}\right)$ and the energy of the photoexcited carriers is larger than that of the side valley minimums ( $L$ and/or $X)$, the electrons can scatter quickly (on the order of $100 \mathrm{fs}$ ) to these valleys. ${ }^{51}$ The electrons in the side valleys return back slowly (on a time scale $>1 \mathrm{ps)}$ to the $\Gamma$ valley. ${ }^{12,25}$ In this case they may act to reheat the electrons in the $\Gamma$ valley and slow the lattice heating.

In our analysis we will assume that the transfer of energy from electrons to LO phonons, characterized by a characteristic time $\tau_{\text {op, }}$, will lead to an optical phonon population in excess of the equilibrium value. ${ }^{11}$ Fröhlich interaction favors
BZ center phonons, however the maximum wave vector $q_{\max }$ of the LO phonons that interact with the electrons depends on the excess energy of the electrons and the $\mathrm{CB}$ curvature and can be on the order of $10^{7} \mathrm{~cm}^{-1} .^{52}$ The LO phonons decay into acoustic phonons through anharmonic interaction with a characteristic time constant $\tau_{\text {ap }}{ }^{10,32}$ This later interaction can be considered to bring the optical phonons into equilibrium with other lattice phonon modes. ${ }^{11,32}$ Quasiequilibrium distributions for all three systems (electrons, LO phonons, and the lattice) are assumed to be established so that the electrons, optical phonons, and lattice have timedependent temperatures $T_{e}, T_{\mathrm{LO}}$, and $T_{l}$. The temporal and spatial evolutions of the system composed of electrons, LO phonons, and the lattice can be described by a set of three coupled nonlinear partial differential equations

$$
\begin{gathered}
C_{e} \frac{\partial T_{e}}{\partial t}=\frac{\partial}{\partial z}\left[\left(E_{\mathrm{CB}}+2 k_{B} T_{e}\right)\left(D_{a} \frac{\partial N_{e}}{\partial z}\right)\right]+\frac{\partial}{\partial z}\left(K_{e} \frac{\partial T_{e}}{\partial z}\right) \\
-C_{e}\left(\frac{T_{e}-T_{\mathrm{LO}}}{\tau_{\mathrm{op}}}\right)+\left(E_{g}+\frac{3}{2} k_{B} T_{e} H_{e}\right) C_{A} N_{e}^{2}, \\
C_{\mathrm{LO}} \frac{\partial T_{\mathrm{LO}}}{\partial t}=C_{e}\left(\frac{T_{e}-T_{\mathrm{LO}}}{\tau_{\mathrm{op}}}\right)-C_{\mathrm{LO}}\left(\frac{T_{\mathrm{LO}}-T_{l}}{\tau_{\mathrm{ap}}}\right),
\end{gathered}
$$

and

$$
C_{l} \frac{\partial T_{l}}{\partial t}=C_{\mathrm{LO}}\left(\frac{T_{\mathrm{LO}}-T_{l}}{\tau_{\mathrm{ap}}}\right),
$$

where $E_{\mathrm{CB}}$ is the $\mathrm{CB}$ edge, $C_{e}$ is the electronic heat capacity, $C_{\mathrm{LO}}$ is the LO phonon heat capacity, $C_{l}$ is the lattice heat capacity, and $K_{e}$ is the electronic thermal conductivity. ${ }^{33}$ The electronic heat capacity is taken as

$$
C_{e}=\frac{\partial}{\partial T_{e}}\left(\frac{3}{2} N_{e} k_{B} T_{e} H_{e}\right),
$$

where $H_{e}$ is a degeneracy factor which depends on the electron energy and temperature. ${ }^{53}$ The LO phonon heat capacity is calculated by assuming that the phonon occupation number depends only on $E_{\mathrm{LO}}$ and $T_{\mathrm{LO}}$, where $E_{\mathrm{LO}}$ is the LO phonon energy near the BZ zone center [for InSb, $E_{\mathrm{LO}}$ $\approx 0.024 \mathrm{eV}$ (Ref. 34)]; this implies a LO phonon heat capacity that is similar to the Einstein model of heat capacity including only the LO phonon branch. ${ }^{54}$ In Eqs. (6) and (8) the cooling of electrons due to the deformation-potential scattering with acoustic phonons is not taken into account since in polar semiconductors this cooling mechanism is thought to become significant for carrier energies less than $E_{\mathrm{LO}} \cdot{ }^{10}$ Following Ref. 35, the energy-loss rate of electrons to acoustic phonons through deformation-potential scattering is more than a factor of 10 smaller than that needed to increase the lattice temperatures within $10-15$ ps to the values observed in the experiment. Reference 35 describes the relaxation of electrons in a metal through deformation-potential scattering. It assumes a parabolic band and chemical potential equal to the Fermi energy which, depending on $N_{e}$ and $T_{e}$, is typically larger than the quasi-Fermi levels in semiconductors. In this case the Fermi distribution function $f_{e}$ is larger than that of a semiconductor, however since the pho- 
non emission rate is $\propto f_{e}\left(E_{e}-\hbar \omega_{\mathrm{AP}}\right)\left[1-f_{e}\left(E_{e}\right)\right]$, with $\hbar \omega_{\mathrm{AP}}$ being the acoustic phonon energy (typically, $<10 \mathrm{meV}$ ), the difference in chemical potentials does not have a large impact on the phonon emission rate.

The left-hand side (lhs) of Eq. (6) represents the rate of change of the energy density of the electron system. The first two terms on the right-hand side (rhs) of Eq. (6) represent the rate of change of the electronic energy density due to diffusion, derived from the relaxation-time approximation of the Boltzmann equation. ${ }^{33,36}$ The third term describes the rate of energy density transfer from electrons to the LO phonons. The emission of LO phonons takes place within hundreds of femtoseconds, but the decay of phonon population has been observed to be several picoseconds: ${ }^{11,30}$ this decay time is long enough that a large nonequilibrium phonon population is created within 1-2 ps after the excitation. Based on this observation we assume that the term describing the rate of energy transfer to the LO phonon system is of this form. The fourth term describes the rate of energy density given to the electron system by Auger heating. In every Auger recombination event, the recombination energy $\sim E_{g}+(3 / 2) k_{B} T_{e} H_{e}$ is transferred to another electron in the $\mathrm{CB} .{ }^{37}$ The energy density given to the electron system is written as a product of the recombination energy and Auger recombination rate.

In Eq. (7) the lhs describes the rate of change of the energy density of the LO phonon population; the first term on the rhs represents the rate of energy density LO phonons gain from the electrons and the second term represents decay into acoustic phonons. The LO phonons have a small dispersion and thus small group velocity. Taking a dispersion of 4 meV over $\Delta q=\pi / a$, where $a=6.479 \AA$ is the lattice constant (Ref. 34), the group velocity $v_{\mathrm{LO}} \approx 1.25 \times 10^{5} \mathrm{~cm} / \mathrm{s}$. Assuming a LO phonon lifetime $\tau_{\mathrm{ap}}=9 \mathrm{ps}$, we obtain a propagation length $v_{\mathrm{LO}} \tau_{\mathrm{ap}} \approx 11 \mathrm{~nm}$, which is about $1 / 8$ of the laser penetration depth. We can conclude that LO phonons are trapped in the photoexcited region and do not propagate significantly.

In Eq. (8), the lhs describes the rate of change of the energy density of the lattice, whereas the rhs represents the rate of energy density which the lattice gains from optical phonons. Diffusive thermal transport during the first few picoseconds ( $\lesssim 15 \mathrm{ps}$ ) is neglected because the estimated diffusion length is about 1/7 of the laser penetration depth.

By solving Eqs. (2) and (3) we obtain the initial density profile for Eq. (4) (see Fig. 2). The solution of Eq. (4) is used then in Eqs. (6)-(8). The initial electron temperature is taken from Eq. (5) with $\Delta E_{e}=\hbar \omega-E_{g} \approx 1.38 \mathrm{eV}$ and the initial LO phonon and lattice temperature are $300 \mathrm{~K}$. The crystal surface is assumed to be impermeable for carriers and carrier energy (i.e., at $z=0, \partial N_{e} / \partial z=0$, and $\partial T_{e} / \partial z=0$ for all times). $D_{a}$ and $E_{\mathrm{CB}}$ are taken as constant and the degeneracy factor $H_{e}=1 .^{55}$ Figure 3 shows the evolution of electron, phonon, and lattice temperatures for a laser fluence of $5 \mathrm{~mJ} / \mathrm{cm}^{2}$, $\tau_{\mathrm{op}}=2 \mathrm{ps}$, and $\tau_{\mathrm{ap}}=6 \mathrm{ps}$. The peak in electron temperature is caused by Auger recombination that heats the electron system through the term $\sim T_{e}$ (cf. Eq. (6)). The LO phonon temperature increases over a time $\sim \tau_{\text {op }}$ to a maximum value of about $1100 \mathrm{~K}$. Although this maximum value of $T_{\mathrm{LO}}$ exceeds the lattice melting temperature $T_{m}=820 \mathrm{~K}$, the relatively high average frequency of LO phonons means that the actual magnitude of the average mean-square displacement

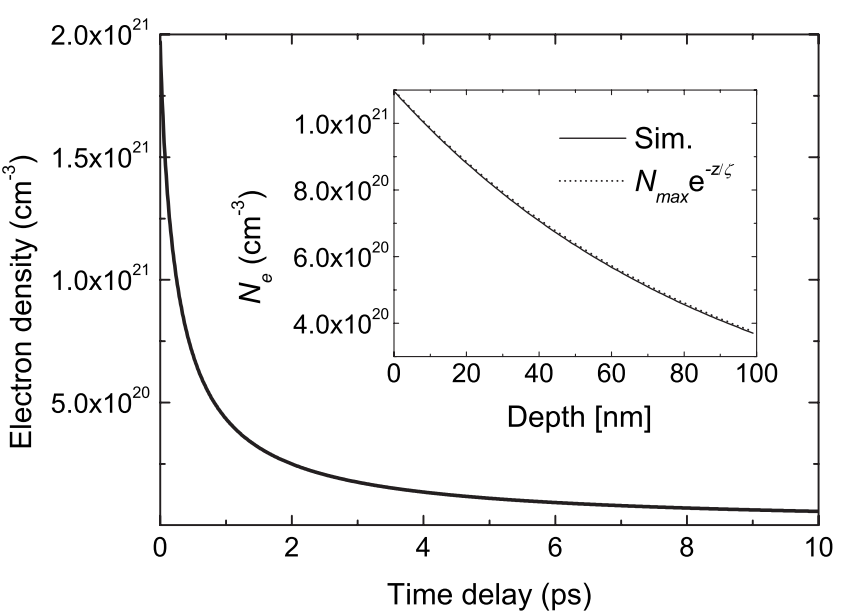

FIG. 2. Evolution of carrier density computed using Eq. (4). Inset: Spatial distribution of carrier density at the end of laser pulse computed using Eqs. (2) and (3) compared to the analytic expression $N_{\max } e^{-z / \zeta}$. This distribution is considered as an initial condition for Eq. (4). The initial condition for Eq. (3) is the equilibrium carrier concentration at room temperature $\left(2 \times 10^{16} \mathrm{~cm}^{-3}\right)$. The temporal profile of the laser intensity is a Gaussian of $120 \mathrm{fs}$ FWHM.

of atoms is considerably lower than it would be if the lattice reached this temperature. We estimate using the equipartition theorem that this temperature corresponds to a mean-square amplitude of atomic vibrations that is $3.9 \%$ of the nearestneighbor distance $(2.793 \AA)$ well below the Lindemann melting criterion $(\sim 10 \%$ of the nearest-neighbor distance). ${ }^{38,39}$ The lattice temperature, on the other hand, increases to a maximum value of about $500 \mathrm{~K}$.

To avoid the computational difficulty of fitting the data to a strain wave arising from the thermal stress derived from an exact solution of Eqs. (6)-(8), we follow Ref. 3 and observe that the general shape of the lattice temperature is well described by an exponential function of the form

$$
T_{l}(t)=T_{0}+\Delta T_{l}\left(1-e^{-t / \tau}\right) .
$$

The rise time $\tau$, hereafter referred to as lattice heating time, depends strongly on the phonon decay time $\tau_{\mathrm{ap}}$. Figure 4

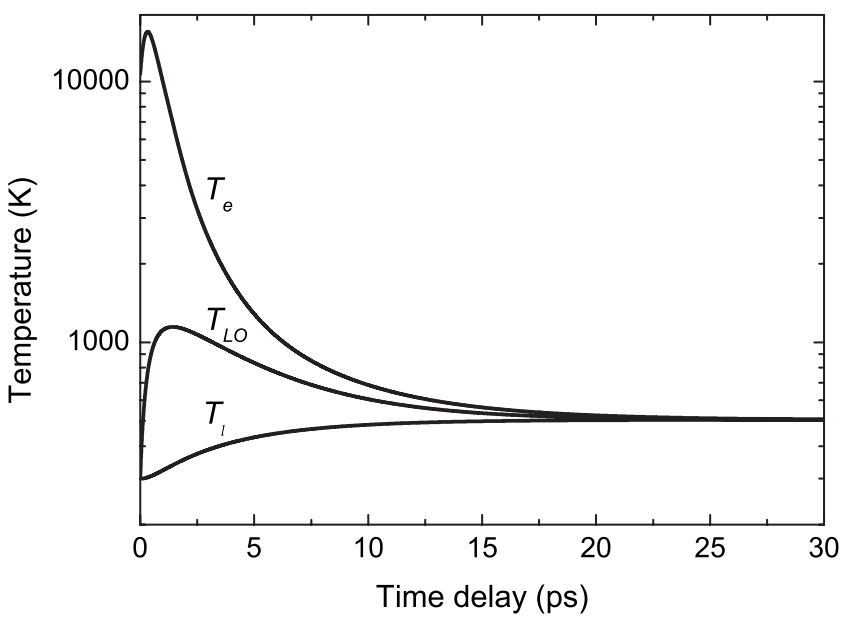

FIG. 3. The dependence of electron, optical phonon and lattice temperatures computed using Eqs. (6)-(8). 


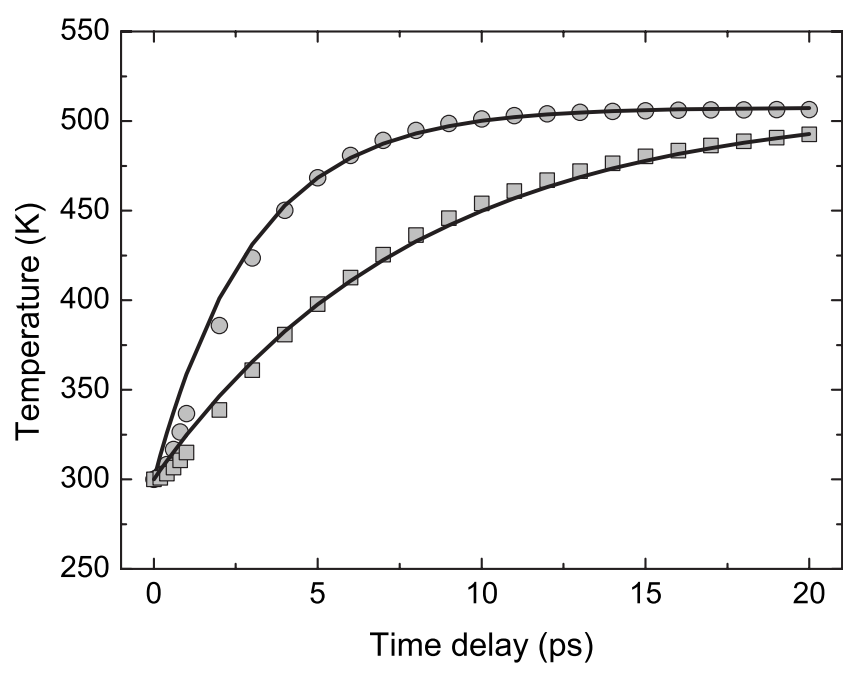

FIG. 4. Approximation of calculated lattice temperature profiles (circles: $\tau_{\mathrm{ap}}=3 \mathrm{ps}$, squares: $\tau_{\mathrm{ap}}=8 \mathrm{ps}$ ) with a function of the form (10) for $\tau=3$ and $8 \mathrm{ps}$, respectively.

compares lattice temperatures computed using Eq. (8) corresponding to optical phonon decay times $\tau_{\text {ap }}$ of 3 and 8 ps with profiles of functional form (10) with rise times $\tau$ of 3 and $8 \mathrm{ps}$, respectively.

\section{B. Strain waves}

The transfer of energy to the lattice leaves the lattice in a highly stressed state, which is eventually relieved by undergoing thermal expansion. Following excitation, the lattice moves toward the new equilibrium state (corresponding to an expanded state) but overshoots and coherently oscillates over a range of frequencies, giving rise to the coherent acoustic pulse, a strain wave. ${ }^{3}$

Thomsen et $a l .{ }^{1}$ presented a thermoelastic model of strain which describes the generation and propagation of a laserinduced coherent strain pulse, hereafter referred to as the Thomsen model. In this model, an ultrafast laser pulse is absorbed and deposits a significant amount of energy near the crystal surface. If the electron-phonon relaxation time is extremely fast (i.e., the lattice is heated instantaneously), this absorption will generate an instantaneous thermal stress

$$
\sigma_{\mathrm{th}}=-3 \beta B \Delta T_{l}(z),
$$

with

$$
\Delta T_{l}(z)=(1-R) \frac{F}{\zeta C_{l}} \exp \left(-\frac{z}{\zeta}\right),
$$

where $\beta$ is the linear-expansion coefficient, $B$ is the bulk modulus, $R$ is the reflectivity of the sample, $F$ is the laser fluence, $C_{l}$ is the lattice heat capacity, and $\zeta$ is the laser penetration depth. For InSb, $\beta=4.7 \times 10^{-6}$ (Ref. 40), $B$ $=46 \mathrm{GPa}$ (Ref. 26), $C_{l}=0.832 \times 10^{6}$ (Ref. 40), and $\zeta$ $\approx 92 \mathrm{~nm}$ [at $800 \mathrm{~nm}$ (Ref. 19)].

With thermal stress of the form in Eq. (11) which assumes an instantaneous conversion of the laser energy to heat, the laser-induced strain wave $\eta(z, t)$ is ${ }^{1}$

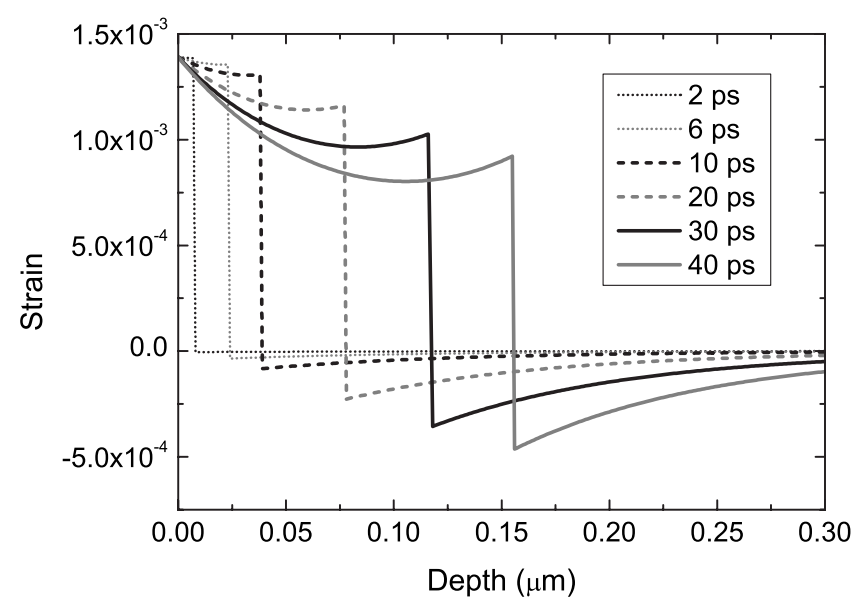

FIG. 5. Laser-generated strain waves at times $t=2,6,10,20,30$, and 40 ps after excitation. The strain waves are calculated using Eq. (13) for InSb at a laser fluence of $3 \mathrm{~mJ} / \mathrm{cm}^{2}$.

$$
\begin{aligned}
\eta(z, t)= & 3(1-R) \frac{F \beta B}{\rho v^{2} \zeta C_{l}}\left\{\exp (-z / \zeta)\left[1-\frac{1}{2} \exp (-v t / \zeta)\right]\right. \\
& \left.-\frac{1}{2} \exp (-|z-v t| / \zeta) \operatorname{sgn}(z-v t)\right\}
\end{aligned}
$$

where $\rho$ is the mass density and $v$ is the longitudinal sound velocity. For InSb, $\rho=5770 \mathrm{~kg} / \mathrm{m}^{3}$ (Ref. 26) and $v$ $=3900 \mathrm{~m} / \mathrm{s}^{6}$ Equation (13) represents a lattice strain wave. The lattice strain profile is shown in Fig. 5 for six different times. It is seen that the resulting lattice motion corresponds to an acoustic pulse propagating into the solid at the velocity of sound. The pulse consists of a region of expansion (or positive strain) followed by a region of compression (negative strain).

In a more realistic model, one needs to consider the fact that the excitation energy initially placed into the carrier system is not transferred instantaneously to the lattice. To incorporate the time needed for excitation energy to be transferred to the lattice, the thermal stress is written in the form

$$
\sigma_{\text {th }}(z, t)=-3 \beta B\left[T_{l}(z, t)-T_{0}\right],
$$

where $T_{l}(z, t)$ is the lattice temperature and $T_{0}=300 \mathrm{~K}$.

Using the arguments in Sec. III A that the lattice temperature increase over the energy-transfer time is almost (with the largest discrepancy not more than $5 \%$ ) of the functional form given by Eq. (10), the thermal stress is written in the form

$$
\sigma_{\mathrm{th}}(z, t)=-3 \beta B[1-\exp (-t / \tau)] T_{l}^{\mathrm{eq}} \exp (-z / \zeta),
$$

where $T_{l}^{\mathrm{eq}}$ is the lattice temperature when the equilibrium with carriers is reached. Here, heat conduction is neglected. The shape of the strain wave is determined by the value $D_{l} /(\zeta v)$, where $D_{l}$ is the thermal diffusion coefficient. For InSb, $D_{l}=0.16 \mathrm{~cm}^{2} / \mathrm{s}$ and $D_{l} /(\zeta v)=0.04$, and according to Ref. 1 , the neglect of heat conduction makes only a small change in the wave shape. However, the magnitude of the strain (especially in the incoherent part) near the target surface will be larger than that where heat conduction is con- 
sidered because the laser-deposited heat is trapped in the excitation region. The difference in magnitudes of the strain with and without consideration of heat conduction depends upon the time delay $\Delta t$ between the pump (laser) and the probe (x rays). For $\Delta t<40 \mathrm{ps,} \mathrm{this} \mathrm{difference} \mathrm{will} \mathrm{be} \mathrm{less}$ than $12 \%$, while in time range $40-80 \mathrm{ps}$, up to $27 \%$. For $\Delta t>500 \mathrm{ps}$, heat conduction has a large effect on the shape of the strain wave. ${ }^{4}$

Such a functional form of the thermal stress was first used in Ref. 3 with $\tau$ representing the phenomenological electronphonon coupling time. There, an analytic expression for the coherent part of the strain wave has been given. In this work $\tau$ represents the LO phonon decay time (cf. Fig. 4), and with thermal stress of the form given by Eq. (15) the full strain wave (coherent+incoherent parts) is obtained which in the limit $\tau \rightarrow 0$ reproduces the Thomsen strain wave. By using Eq. (15) and Eqs. (4)-(6) in Ref. 1 one can obtain an analytical expression for the laser-induced displacements $u(z, t)$ and subsequently for the strain $\eta(z, t)=\partial u(z, t) / \partial z$. The solutions are $^{56}$

(i) $z<v t$

$$
\begin{gathered}
u(z, t)=G_{1}(z, t)+\frac{1}{2 v} G_{2}(z, t), \\
G_{1}(z, t)=A_{1}\left[1-e^{-v / \zeta(t-z / v)}\right]\left[1-e^{-1 / \tau(t-z / v)}\right], \\
G_{2}(z, t)=A_{2} \frac{\zeta^{2} e^{-2 z / \zeta}}{v}\left\{\frac { ( e ^ { 2 z / \zeta } - 1 ) } { \zeta - v \tau } \left[\zeta\left(1-e^{(z-v t) / \zeta}\right)\right.\right. \\
\left.\left.+v \tau\left(e^{(z-v t) /(v \tau)}-1\right)\right]+\left(e^{z / \zeta}-1\right)^{2}\right\}+A_{2} \frac{\zeta^{2} e^{-2 z / \zeta}}{v} \\
\times \frac{v \tau e^{-t / \tau}\left\{2 v \tau e^{z / \zeta}-e^{z /(v \tau)}\left[\zeta+v \tau+e^{2 z / \zeta}(v \tau-\zeta)\right]\right\}}{\left(v^{2} \tau^{2}-\zeta^{2}\right)},
\end{gathered}
$$

(ii) $z>v t$

$$
\begin{aligned}
u(z, t)= & A_{2} \zeta^{2} e^{-z / \zeta}\left\{\frac{\cosh (v t / \zeta)-1}{v^{2}}\right. \\
& \left.+\frac{\tau\left[v \tau e^{-t / \tau}-v \tau \cosh (v t / \zeta)+\zeta \sinh (v t / \zeta)\right]}{v\left(v^{2} \tau^{2}-\zeta^{2}\right)}\right\},
\end{aligned}
$$

where

$$
\begin{gathered}
A_{1}=-\frac{3 \beta B \zeta}{v^{2} \rho} \Delta T_{l}^{\mathrm{eq}}, \\
A_{2}=\frac{3 \beta B}{\zeta \rho} \Delta T_{l}^{\mathrm{eq}} .
\end{gathered}
$$

If we use $\Delta T_{\mathrm{eq}}=(1-R) F /\left(\zeta C_{l}\right)$ in Eqs. (18) and (19) and take the limit $\tau \rightarrow 0$, we retrieve the traditional Thomsen strain profile. Calculated strain profiles using Eqs. (16)-(19) are shown in Fig. 6. The effect of the heating time $\tau$ is evident. We see that the strain near the surface $(z=0)$ increases over the time $\tau$, in contrast to Fig. 5 where it is almost time

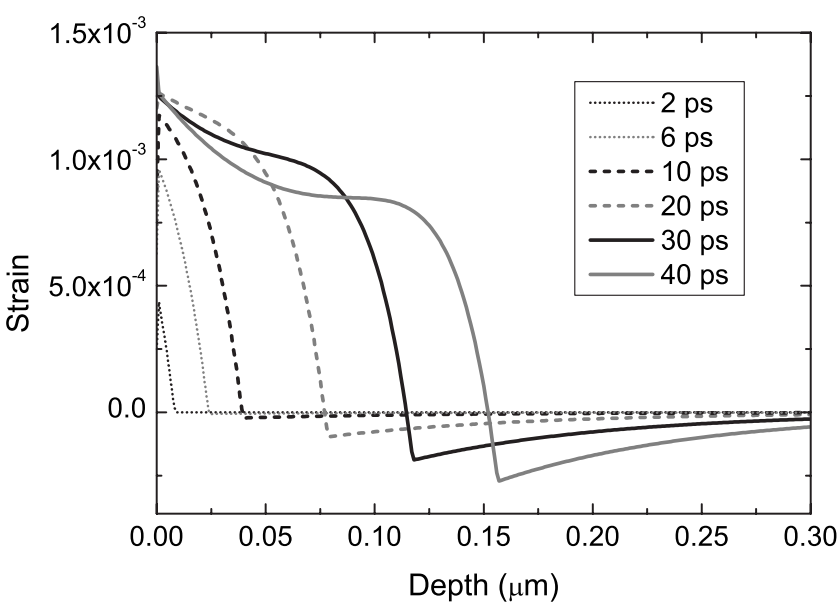

FIG. 6. Laser-generated strain waves at times $t=2,6,10,20,30$, and $40 \mathrm{ps}$ after excitation using a heating time $\tau=10 \mathrm{ps}$. The strain waves are calculated using Eqs. (16)-(19) for InSb at laser fluence of $3 \mathrm{~mJ} / \mathrm{cm}^{2}$.

independent, and the boundary between the expansive and compressive regions is smoothed. Figure 7 shows the strain wave profile $20 \mathrm{ps}$ after excitation for different heating times $\tau$.

\section{X-RAY DIFFRACTION IN LASER-EXCITED CRYSTALS}

$\mathrm{X}$-ray diffraction in the presence of laser-induced strain is calculated using the Takagi-Taupin (TT) dynamical theory for the depth-dependent strain gradients. ${ }^{14}$ This theory has been successfully applied to model x-ray diffraction from coherent acoustic phonons. ${ }^{2,3,6,7,11,41}$ Within this theory the differential equation for the ratio of the complex field amplitudes of the diffracted x-rays $D_{h}$ and incident x-rays $D_{0}$ can be written as ${ }^{14}$

$$
\frac{d \xi}{d z}=\frac{\pi i}{\Lambda}\left[\xi^{2}-2 S\left(\gamma_{h}\right) \beta_{\Delta \theta} \xi-\frac{\left|\gamma_{h}\right|}{\gamma_{h}}\right],
$$

where

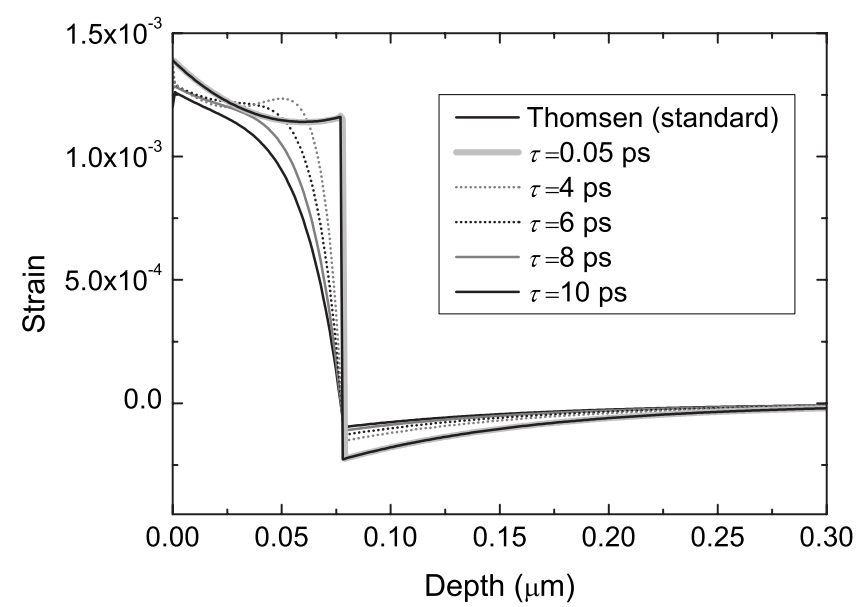

FIG. 7. Laser-generated strain wave 20 ps after excitation using heating times $\tau=0.05,4,6,8$, and $10 \mathrm{ps}$. The strain waves are calculated using Eqs. (16)-(19) for InSb at laser fluence of $3 \mathrm{~mJ} / \mathrm{cm}^{2}$. 


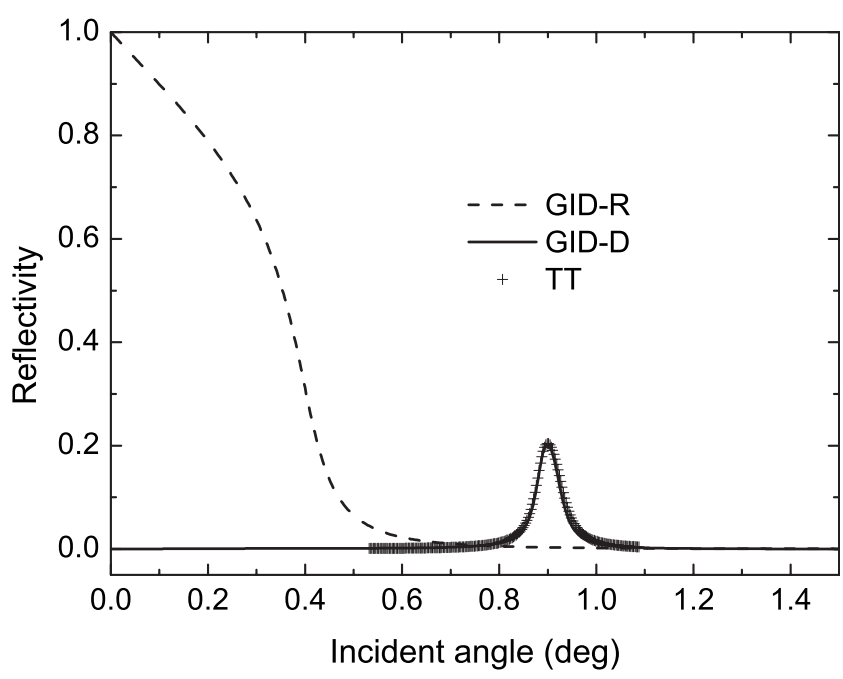

FIG. 8. Comparison of the unperturbed rocking curve for InSb (5.9 $\mathrm{keV}, 111$ reflection, $\alpha=15.5^{\circ}$ ) calculated using the TakagiTaupin theory and the thick crystal rocking curve [grazing incidence $\mathrm{x}$-ray diffraction (GID-D)] and x-ray reflection (GID-R) calculated using the theory of $\mathrm{x}$-ray diffraction under grazing incidence conditions.

$$
\begin{gathered}
\xi=\frac{1}{S(P)} \sqrt{\frac{\left|\gamma_{h}\right|}{\gamma_{0}} \frac{\chi_{\bar{h}}^{-}}{\sqrt{\chi_{h} \chi_{\bar{h}}}} \frac{D_{h}}{D_{0}}}, \\
\Lambda=\frac{\lambda \sqrt{\gamma_{0}\left|\gamma_{h}\right|}}{|P| \sqrt{\chi_{h} \chi_{h}^{-}}} \\
\beta_{\Delta \theta}=\frac{\sqrt{\gamma_{0} /\left|\gamma_{h}\right|}}{|P| \sqrt{\chi_{h} \chi_{h}^{-}}}\left[(\Delta \theta+C \eta) \sin 2 \theta_{B}-\frac{1}{2} \chi_{0}\left(\frac{\gamma_{h}}{\gamma_{0}}-1\right)\right], \\
C=\cos ^{2} \alpha \tan \theta_{B}+\sin \alpha \cos \alpha
\end{gathered}
$$

and

$$
\chi_{h}=\frac{r_{0} \lambda^{2} F_{h}}{\pi V} .
$$

Here $\Delta \theta=\theta-\theta_{B}, \gamma_{0(h)}$ are the direction cosines of the incident (diffracted) beam, $\lambda$ is the x-ray wavelength, $\eta(z, t)$ is the strain, $F_{h}$ is the structure factor, $r_{0}$ is the classical electron radius $\left(r_{0}=2.818 \times 10^{-15} \mathrm{~m}\right), V$ is the volume of the unit cell, $S(x)$ is the sign function, and $P$ is the polarization factor. In this experiment the $\mathrm{x}$ rays are $s$ polarized and $P$ $=1$. Equation (20) is solved analytically (see the Appendix) under the condition that deep in the crystal $\left(z=z_{m}\right)$ where the strain vanishes $\xi\left(z_{m}\right)=\xi_{p}$, where $\xi_{p}$ is the value of $\xi$ for a perfect crystal. ${ }^{14,42}$ From Eq. (21) one can find $D_{h} / D_{0}$ and finally the rocking curve of the crystal

$$
R=\frac{\left|\gamma_{h}\right|}{\gamma_{0}}\left|\frac{D_{h}(z=0)}{D_{0}(z=0)}\right|^{2} .
$$

Figure 8 compares the unperturbed rocking curves predicted by Eq. (26) to the rocking curve of a thick crystal predicted

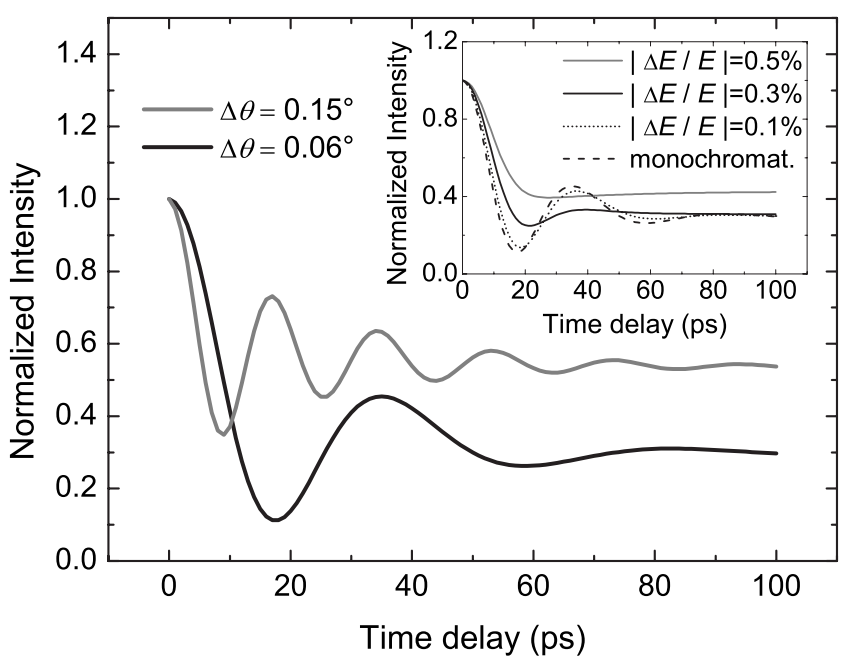

FIG. 9. Time evolution of the normalized diffracted intensity calculated using Eqs. (20)-(26) at two different positions on the rocking curve, $\Delta \theta_{B}=0.06^{\circ}$ and $0.15^{\circ}$. The laser-induced strain is calculated using Eqs. (16)-(19) with $\tau=5$ ps and $T_{l}^{\mathrm{eq}}=240 \mathrm{~K}$. Inset: The effect of the $\mathrm{x}$-ray bandwidth on the time evolution of the diffracted intensity. The bandwidth of the beam is taken into account by convolving the calculated rocking curves by a Gaussian function of FWHM given by Eq. (28).

by the dynamical theory of x-ray diffraction under grazing incidence conditions. ${ }^{43}$ The rocking curve computed by the grazing incidence $\mathrm{x}$-ray diffraction theory compares well to that using TT theory indicating that the negligence of x-ray reflection does not have any impact on the rocking curve.

The laser-induced strain wave $\eta(z, t)$ is a superposition of coherent acoustic phonons with wave vectors $q$ approximately centered about the inverse of the laser penetration depth. ${ }^{6,41}$ Due to the coherent acoustic phonons of wave vector $q$ the time-resolved x-ray diffraction intensity will oscillate with an angular frequency $\omega$ given by ${ }^{41}$

$$
\omega \approx v q \approx \frac{v \Delta \theta_{B}\left|\mathbf{G}_{h}\right|}{\tan \theta_{B} \cos \alpha+\sin \alpha},
$$

where $\Delta \theta_{B}$ is the angular position off the Bragg peak and $\mathbf{G}_{h}$ is the reciprocal-lattice vector. Equation (27) indicates that by varying $\Delta \theta_{B}$ different phonon modes with frequencies $\omega\left(\Delta \theta_{B}\right)$ can be observed. Figure 9 shows the normalized time-dependent X-ray diffracted intensity for $\Delta \theta_{B}=0.06^{\circ}$ and $0.15^{\circ}$. The time evolution of the diffracted intensity has basically two origins. First, the reduction of the intensity is due to a shift of the rocking curve. Second, this reduction is superimposed by an interference of $\mathrm{x}$-rays originating from two sources: (a) the bulk x-ray diffraction and (b) the diffraction from the strain wave. The diffraction from the strain wave leads to the oscillations in the time-dependent diffracted intensity with periods $T=2 \pi / \omega=48$ and $18 \mathrm{ps}$, respectively, which are comparable to those predicted by Eq. (27), which are 49 and 20 ps. The visibility of the temporal oscillations is reduced with increasing bandwidth of the $\mathrm{x}$ rays. ${ }^{41}$ The inset of Fig. 9 shows the effect of the X-ray bandwidth on the time evolution of the diffracted intensity. The oscillation amplitude of the time-dependent diffracted inten- 


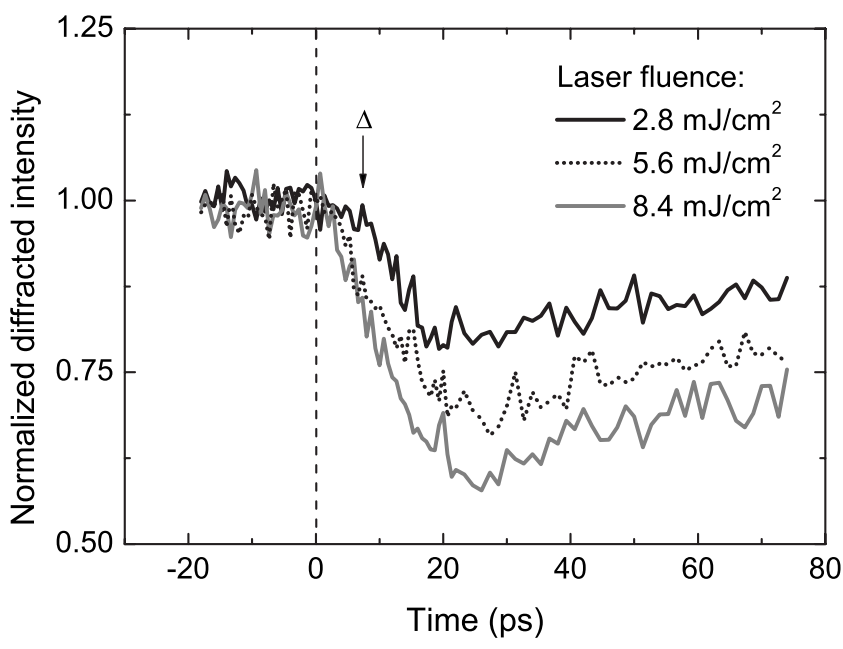

FIG. 10. Measured time-dependent normalized diffracted intensities from InSb with step size of 670 fs for laser fluences 2.8, 5.6, and $8.4 \mathrm{~mJ} / \mathrm{cm}^{2}$. The time scans are measured at $5.9 \mathrm{keV}$ (2.101 ̊), 111 reflection, $\alpha \approx 15.5^{\circ}$.

sity decreases with the increase in the bandwidth of the $\mathrm{x}$ rays.

\section{EXPERIMENTAL RESULTS AND DISCUSSION}

In this section we describe the effects of carrier-lattice thermalization on laser-induced strain waves. In the experiment, we have measured the evolution of x-ray diffraction intensity as a function of laser fluence below the damage threshold of $10-11 \mathrm{~mJ} / \mathrm{cm}^{2}{ }^{6,44,57}$ Figure 10 shows the timedependent diffracted intensity measured at $+0.06^{\circ}$ from the unperturbed Bragg peak with fluences of $(2.8 \pm 0.6) \mathrm{mJ} / \mathrm{cm}^{2}, \quad(5.6 \pm 1.2) \mathrm{mJ} / \mathrm{cm}^{2}, \quad$ and $(8.4 \pm 1.8) \mathrm{mJ} / \mathrm{cm}^{2}$. Time zero has been determined by using x-ray diffraction to probe laser-induced coherent optical phonons in bulk bismuth. ${ }^{13}$ The effective time resolution is $195 \pm 25$ fs FWHM including time drifts of 70 fs measured for several days. This enables multishot data accumulation during extended consecutive time scans.

The fluence-dependent effects are immediately apparent. First, the value of the intensity minimum decreases with increasing fluence. Second, at the lowest fluence $\left(\sim 2.8 \mathrm{~mJ} / \mathrm{cm}^{2}\right)$ over the first $15 \mathrm{ps}$ following the laser excitation, the rate of decrease in the diffracted signal is slower than at higher fluences (see the arrow identified with $\Delta$ ).

In order to extract physical information from the data presented in Fig. 10, we use the model presented in Sec. III. The $\mathrm{X}$-ray diffraction is simulated by using Eqs. (20)-(26). The bandwidth of the double multilayer monochromator $\Delta E / E$ is taken into account by convolving the calculated rocking curves by a Gaussian function of FWHM

$$
\Delta \theta_{\mathrm{BW}}=\left|\frac{\Delta E}{E}\right| \tan \theta .
$$

Oscillations in the time-dependent diffraction intensities shown in Fig. 10 are washed out due to the large bandwidth of the $\mathrm{x}$ rays. The contribution of the Debye-Waller factor in

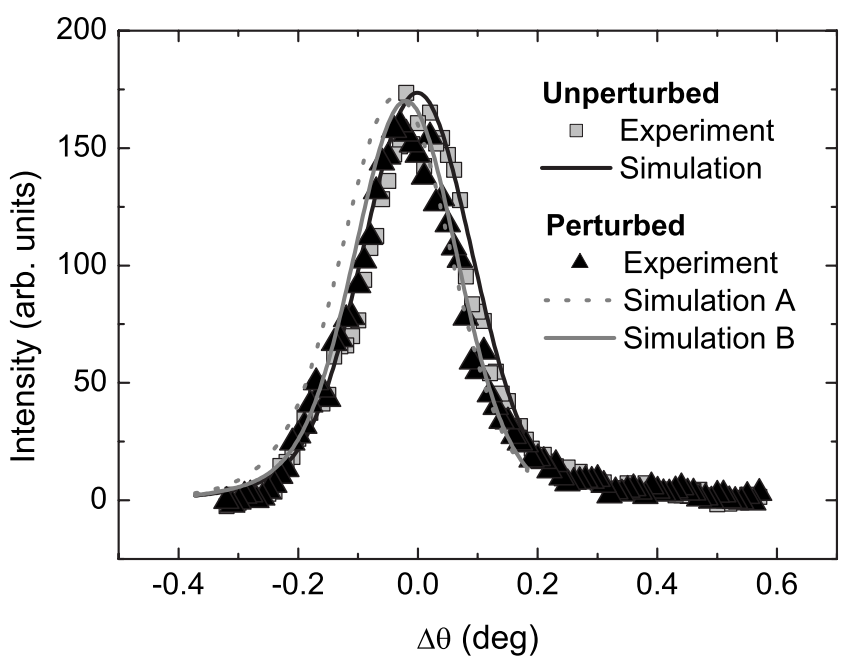

FIG. 11. Comparison between the simulated and the measured rocking curves. The calculated rocking curves are convolved with a Gaussian function corresponding to $\Delta E / E \approx 1.1 \%$. In the perturbed crystal case, the rocking curve is measured at a laser fluence of about $7 \mathrm{~mJ} / \mathrm{cm}^{2}$ and time delay $\Delta t \approx 75 \mathrm{ps}$. In the simulations we have used $F=7 \mathrm{~mJ} / \mathrm{cm}^{2}, \Delta t=75 \mathrm{ps}$, and $\tau=0$ ps. Simulation A does not take into account the effect of heat conduction. Simulation B accounts for heat conduction by assuming 26\% lower surface temperature and $19 \%$ larger penetration depth. These values were estimated by solving Eq. (8) with heat conduction term $D_{l} \partial^{2} T_{l} / \partial z^{2}$ (where $D_{l}$ is the thermal diffusion coefficient) included in the rhs, initial condition $T_{l}(z, t=0)=T_{0} \exp (-z / \zeta)$, and boundary condition $\partial T_{l}(z=0, t) / \partial z=0$.

the diffracted intensity is neglected. Over the temperature range up to $600 \mathrm{~K}$ and for the mean-square displacement up to $8 \%$ of the nearest-neighbor distance the decrease in the diffracted intensity due to the Debye-Waller effect for the 111 reflection is $\$ 4 \%$. Figure 11 shows the comparison between the simulated and measured rocking curves for the unperturbed (zero strain) and perturbed crystals. Once the unperturbed rocking curve is reproduced, we simulate rocking curves with strain profiles computed using Eqs. (16)-(19) by changing only the fluence $F$ and the heating time $\tau$, whereas the other parameters are held fixed. The discrepancies in the small-angle side $(\sim 26 \%)$ are due to the neglect of heat conduction in the calculation of the strain wave. In this case the strain will be larger than that where heat conduction is considered because the laser-deposited heat is trapped in the excitation region; the rocking curve is shifted more in the small-angle side and is broader than that with heat conduction included. Since the small-angle side of the rocking curve is more sensitive to thermal expansion that is proportional to the lattice temperature, the discrepancies due to the neglect of heat conduction are pronounced more there. During the first 20 ps following the laser excitation when the effect of the lattice heating time is large, the discrepancies on the positive angle side of the rocking curve are smaller than $4 \%$ whereas on the negative angle side up to $7 \%$. For time delays between 20 and 70 ps the discrepancies due to the neglect of heat conduction are about $5 \%$ in the positive angle side and up to $22 \%$ on the negative (small) angle side.

As shown in Fig. 12, the simulations predict a faster intensity drop for $\tau=0$. In the instantaneous heating limit the 


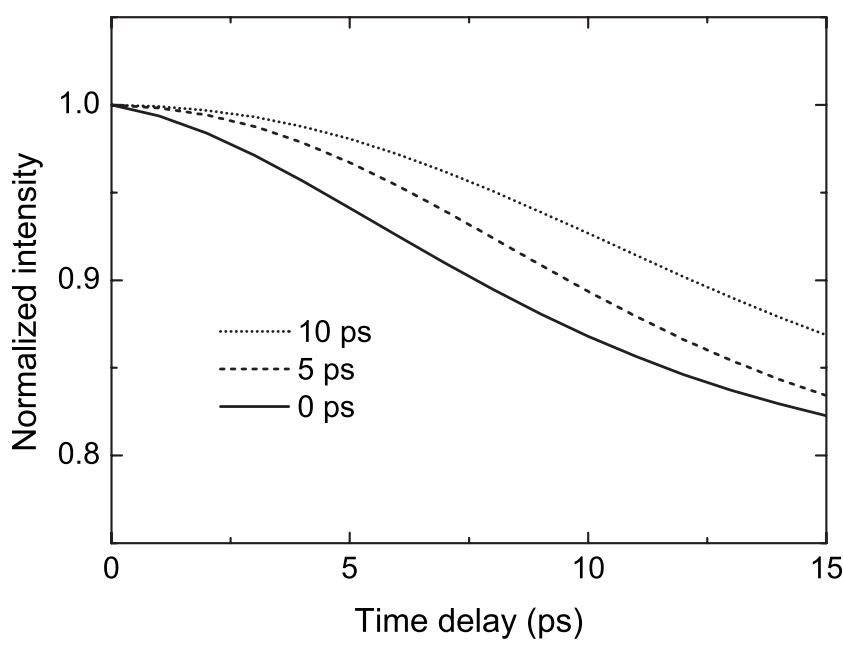

FIG. 12. Calculated time-dependent diffracted intensity (at $+0.06^{\circ}$ relative to the Bragg peak) assuming a strain history calculated using Eqs. (16)-(19), with heating times $\tau=0$, 5, and 10 ps.

strain is initially large (cf. Fig. 5). As the strain wave propagates inside the crystal, the $\mathrm{x}$ rays probe a large-amplitude strained region with a thickness that increases with the speed of sound. On the other hand, assuming a finite lattice heating time $\tau$, the strain which is initially zero increases during the time $\tau$ as shown in Fig. 6. In this case the $\mathrm{x}$ rays probe a strained region which increases with the speed of sound, but whose amplitude increases with time. This gives rise to a slower drop in the x-ray intensity compared to the instantaneous heating limit.

Experimental results are compared to simulations in Figs. 13 and 14. In the simulations, the lattice temperature increase in the crystal surface predicted by Eq. (8) for fluences 2.8, 5.6 , and $8.4 \mathrm{~mJ} / \mathrm{cm}^{2}$ is 158,240 , and $295 \mathrm{~K}$, respectively. The expected excitation densities at these fluences are 1.1 $\times 10^{21} \mathrm{~cm}^{-3}, 2.3 \times 10^{21} \mathrm{~cm}^{-3}$, and $3.3 \times 10^{21} \mathrm{~cm}^{-3}$. In Fig. 13 the measured diffracted intensity for the fluence of $2.8 \mathrm{~mJ} / \mathrm{cm}^{2}$ is compared to simulations assuming instanta-

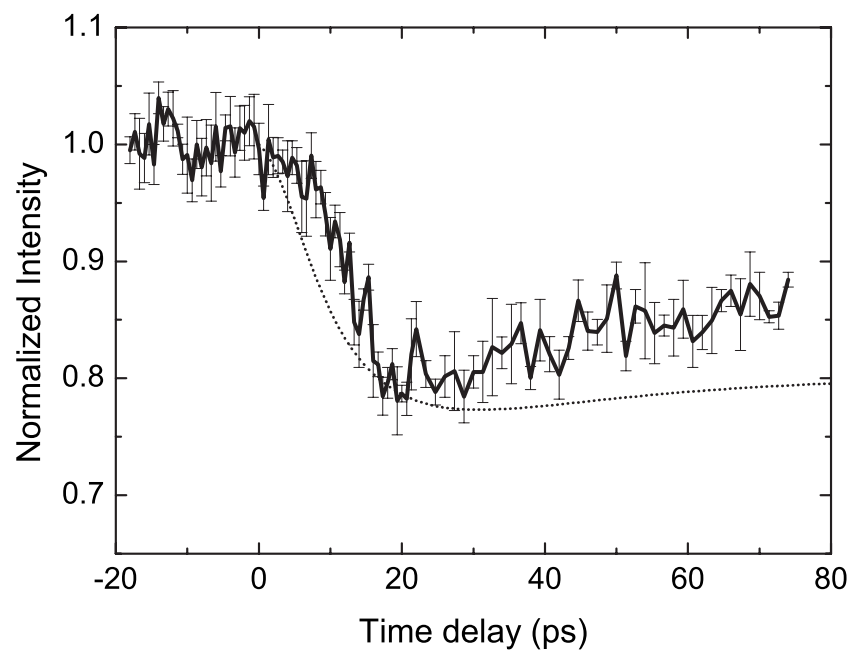

FIG. 13. Comparison of the measured time-dependent normalized diffracted intensity (solid line) to simulations assuming strain history given by using Eqs. (16)-(19) with $\tau=0$ ps (dotted line).

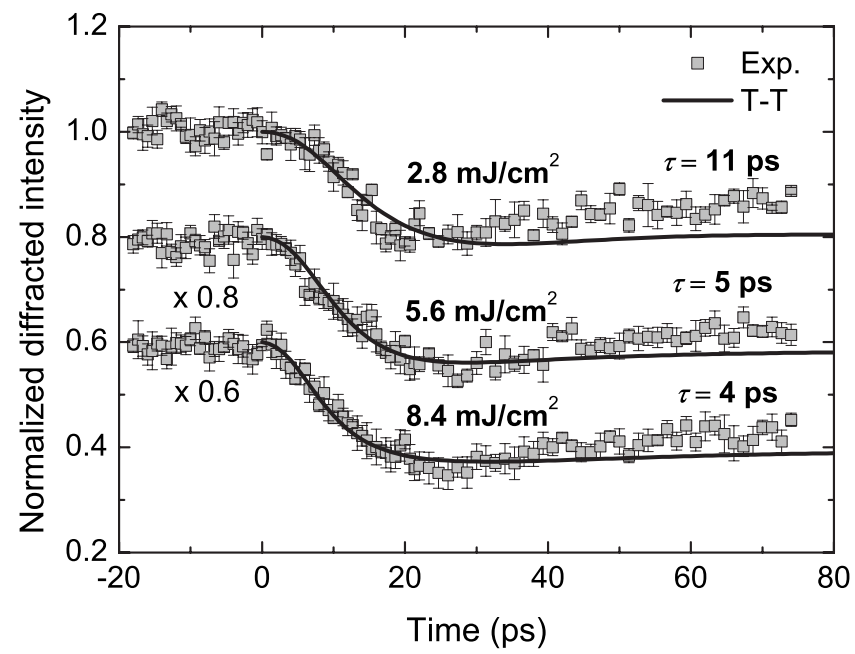

FIG. 14. Comparison of measured time-dependent normalized diffracted intensities shown in Fig. 10 to simulations assuming strain history given by using Eqs. (16)-(19).

neous heating of the lattice, i.e., $\tau=0$ ps. During the first 15-18 ps, the instantaneous heating time predicts a much faster drop in intensity than the measured one. Assuming a lattice heating time $\tau=11 \pm 4 \mathrm{ps}$, simulations predict a slow decrease in the diffracted intensity during this time in agreement with the experimental results (see Fig. 14) ${ }^{58}$ For higher fluences of 5.6 and $8.4 \mathrm{~mJ} / \mathrm{cm}^{2}$, a good agreement between the experiment and calculations is found for $\tau=5 \pm 2$ and $4 \pm 1.5$ ps, respectively. For time delays $>20$ ps the measured diffracted intensity is about $4 \%$ larger than that predicted by the simulations. This discrepancy is mainly due to the omission of heat conduction in the calculation of the strain profiles that results in larger strains and thus smaller diffracted intensities.

Reproduction of the slow drop of the diffracted intensity by including the heating time in the strain wave suggests that this effect is a signature of the phonon dynamics subsequent to carrier energy relaxation as discussed in Sec. III A, since the lattice heating time is largely dependent on the optical phonon decay time (cf. Fig. 4). As the population of LO phonons increases via energy transfer from the carriers, the atoms undergo anharmonic motion. This anharmonicity couples the LO phonons to acoustic phonons. In a similar way, Chin et al. ${ }^{11}$ described the delay on the onset of the time-dependent diffracted intensity from InSb. At a carrier density above $10^{21} \mathrm{~cm}^{-3}$, they were able to describe their observed effect by assuming a 2 ps LO emission time and 7 ps acoustic phonon generation time (i.e., LO phonon decay time). Since the heating time $\tau$ depends strongly on the phonon decay time, the decrease in the lattice heating time with increasing laser fluence suggests that the LO phonon decay time decreases with increasing laser fluence (i.e., with increasing excitation energy). A similar effect has been observed by time-resolved Raman studies of phonon lifetimes in $\mathrm{GaN}$ which is a polar semiconductor like InSb. Tsen et $a l .{ }^{46}$ observed that the phonon lifetime of $\mathrm{GaN}$ decreases from 2.5 to $0.35 \mathrm{ps}$ when the $e-h$ density increases from $10^{16}$ to $2 \times 10^{19} \mathrm{~cm}^{-3}$. Although GaN, which is a polar and directgap semiconductor, differs from InSb in terms of band-gap 
energy and band curvatures, the observations of Tsen et al. ${ }^{46}$ support the fact that the LO phonon decay depends strongly on the electron density. Intervalley scattering may also influence the carrier density dependence of the lattice heating time, but this should have the effect of increasing the lattice heating time as the fluence increases, which runs counter to our observations.

The matrix elements of the anharmonic decay rate depend on the phonon occupation number ${ }^{47}$ which increases with increasing LO phonon temperature and thus with increasing laser fluence. This indicates that LO phonon decay rate increases (i.e., the phonon lifetime decreases) with increasing laser fluence (i.e., with increasing electron density) which is in accordance with our observations. However, Matulionis ${ }^{48}$ suggested that the dependence of LO phonon decay time on carrier density can be explained by assuming that plasmons are involved in LO phonon disintegration (LO phonons can emit plasmons in the decay process). Therefore, a more rigorous approach that considers the decay of LO phonons via both acoustic phonons and plasmons is needed to provide a plausible answer regarding the dependence of the LO phonon lifetime on the carrier density or laser fluence. To our knowledge, the mechanism for the decay of LO phonons via acoustic phonons and plasmons has not been worked out so far.

\section{CONCLUSIONS}

In conclusion, we have presented a study of laser-induced strain waves in InSb over an intermediate fluence range $\left(\sim 3-10 \mathrm{~mJ} / \mathrm{cm}^{2}\right)$. We have presented a model that predicts spatiotemporal evolution of strain waves during the lattice heating time. In the instantaneous heating limit the model predicts Thomsen-type strain waves. In the framework of this model and the Takagi-Taupin dynamical theory for the depth-dependent strain gradients we have studied the fluence dependence of the transient $\mathrm{x}$-ray diffraction signal. The net result of this analysis is that the temporal evolution of the diffracted signal indicates that the lattice heating time de- creases with increasing fluence. This implies that the lifetime of optical phonons decreases as the excitation energy increases, similar to previous observations in other polar, direct-band-gap semiconductors. ${ }^{46}$ Although the lifetime of LO phonons in general is dominated by their decay into a pair of acoustic phonons, the density dependence of the LO phonon decay time is not fully understood ${ }^{46}$ and requires further investigations.

\section{ACKNOWLEDGMENTS}

The authors gratefully acknowledge financial support by the European Union in Framework No. I3-JRP3. We thank P. Sondhauss for bringing Ref. 14 to our attention.

\section{APPENDIX}

Analytical solution of Eq. (20) is

$$
\xi(z)=\frac{s \xi_{0}+\left(B \xi_{0}+C\right) \tan \left[s\left(z-z_{0}\right)\right]}{s-\left(A \xi_{0}+B\right) \tan \left[s\left(z-z_{0}\right)\right]},
$$

where

$$
\begin{gathered}
\xi\left(z_{0}\right)=\xi_{0}, \\
A=\frac{\pi i}{\Lambda},
\end{gathered}
$$

$$
\begin{gathered}
B=-\frac{\pi i}{\Lambda} S\left(\gamma_{h}\right) \beta_{\Delta \theta}, \\
C=-\frac{\pi i}{\Lambda} \frac{\left|\gamma_{h}\right|}{\gamma_{h}},
\end{gathered}
$$

and

$$
s=\sqrt{A C-B^{2}} .
$$

Hence the value of $\xi$ at the depth $z$ can be calculated by knowing its value at $z=z_{0}$.
*Present address: Max Planck Advanced Study Group at CFEL/ DESY, Notkestr. 85, 22607 Hamburg, Germany. faton.krasniqi@asg.mpg.de

${ }^{1}$ C. Thomsen, H. T. Grahn, H. J. Maris, and J. Tauc, Phys. Rev. B 34, 4129 (1986).

${ }^{2}$ Ch. Rose-Petruck, R. Jimenez, T. Guo, A. Cavalleri, C. W. Siders, F. Ráksi, J. A. Squier, B. C. Walker, K. R. Wilson, and C. P. J. Barty, Nature (London) 398, 310 (1999).

${ }^{3}$ A. M. Lindenberg, Ph.D. thesis, University of California, 2001.

${ }^{4}$ G. Tas and H. J. Maris, Phys. Rev. B 49, 15046 (1994).

${ }^{5}$ O. B. Wright, Phys. Rev. B 49, 9985 (1994).

${ }^{6}$ A. M. Lindenberg, I. Kang, S. L. Johnson, T. Missalla, P. A. Heimann, Z. Chang, J. Larsson, P. H. Bucksbaum, H. C. Kapteyn, H. A. Padmore, R. W. Lee, J. S. Wark, and R. W. Falcone, Phys. Rev. Lett. 84, 111 (2000).

${ }^{7}$ D. A. Reis, M. F. DeCamp, P. H. Bucksbaum, R. Clarke, E.
Dufresne, M. Hertlein, R. Merlin, R. Falcone, H. Kapteyn, M. M. Murnane, J. Larsson, Th. Missalla, and J. S. Wark, Phys. Rev. Lett. 86, 3072 (2001).

${ }^{8}$ M. F. DeCamp, D. A. Reis, A. Cavalieri, P. H. Bucksbaum, R. Clarke, R. Merlin, E. M. Dufresne, D. A. Arms, A. M. Lindenberg, A. G. MacPhee, Z. Chang, B. Lings, J. S. Wark, and S. Fahy, Phys. Rev. Lett. 91, 165502 (2003).

${ }^{9}$ See, for example, M. F. DeCamp, D. A. Reis, D. M. Fritz, P. H. Bucksbaum, E. M. Dufresne, and R. Clarke, J. Synchrotron Radiat. 12, 177 (2005), and references therein.

${ }^{10}$ J. Shah, Ultrafast Spectroscopyof Semiconductors and Semiconductor Nanostructures (Springer, Berlin, 1996).

${ }^{11}$ A. H. Chin, R. W. Schoenlein, T. E. Glover, P. Balling, W. P. Leemans, and C. V. Shank, Phys. Rev. Lett. 83, 336 (1999).

${ }^{12}$ J. Shah, B. Deveaud, T. C. Damen, W. T. Tsang, A. C. Gossard, and P. Lugli, Phys. Rev. Lett. 59, 2222 (1987). 
${ }^{13}$ P. Beaud, S. L. Johnson, A. Streun, R. Abela, D. Abramsohn, D. Grolimund, F. Krasniqi, T. Schmidt, V. Schlott, and G. Ingold, Phys. Rev. Lett. 99, 174801 (2007).

${ }^{14}$ J. Gronkowski, Phys. Rep. 206, 1 (1991).

${ }^{15}$ R. W. Schoenlein, S. Chattopadhyay, H. H. W. Chong, T. E. Glover, P. A. Heimann, C. V. Shank, A. A. Zholents, and M. S. Zolotorev, Science 287, 2237 (2000).

${ }^{16}$ C. T. Chantler, J. Phys. Chem. Ref. Data 29, 597 (2000).

${ }^{17} \mathrm{See}$, for example, S. K. Sundaram and E. Mazur, Nature Mater. 1, 217 (2002), and references therein.

${ }^{18}$ K. Sokolowski-Tinten and D. von der Linde, Phys. Rev. B 61, 2643 (2000)

${ }^{19}$ D. E. Aspnes and A. A. Studna, Phys. Rev. B 27, 985 (1983).

${ }^{20}$ H. S. Brandi and C. B. de Araújo, J. Phys. C 16, 5929 (1983).

${ }^{21}$ N. W. Ashcroft and N. D. Mermin, Solid State Physics (Saunders, Philadelphia, 1976).

${ }^{22}$ P. M. Fauchet, Phys. Status Solidi B 110, K11 (1982).

${ }^{23}$ H. M. van Driel and J. F. Young, J. Phys. C 15, L31 (1982).

${ }^{24}$ J. F. Young and H. M. van Driel, Phys. Rev. B 26, 2147 (1982).

${ }^{25}$ C. J. Stanton and D. W. Bailey, Phys. Rev. B 45, 8369 (1992).

${ }^{26}$ Semiconductors: Physics of Group IV Elements and III-V Compounds, Landolt-Börnstein, New Series, Group III: Crystal and Solid State Physics Vol. 17, Pt. A, edited by O. Madelung (Springer, Berlin, 1982).

${ }^{27}$ C. V. De Alvarez, J. P. Walter, R. W. Boyd, and M. L. Cohen, J. Phys. Chem. Solids 34, 337 (1973).

${ }^{28}$ Th. Elsaesser, J. Shah, L. Rota, and P. Lugli, Phys. Rev. Lett. 66, 1757 (1991).

${ }^{29}$ J. Shah, Solid-State Electron. 21, 43 (1978).

${ }^{30}$ D. von der Linde, J. Kuhl, and H. Klingenberg, Phys. Rev. Lett. 44, 1505 (1980).

${ }^{31}$ S. S. Prabhu, A. S. Vengurlekar, S. K. Roy, and J. Shah, Phys. Rev. B 51, 14233 (1995).

${ }^{32}$ H. M. van Driel, Phys. Rev. B 19, 5928 (1979).

${ }^{33}$ J. R. Drabble and H. J. Goldsmid, Thermal Conduction in Semiconductors (Pergamon, New York, 1961).

${ }^{34}$ D. L. Price, J. M. Rowe, and R. M. Nicklow, Phys. Rev. B 3, 1268 (1971).

${ }^{35}$ M. I. Kaganov, I. M. Lifshitz, and L. V. Tanatarov, Sov. Phys. JETP 4, 173 (1957).

${ }^{36}$ H. M. van Driel, Phys. Rev. B 35, 8166 (1987).

${ }^{37}$ M. C. Downer and C. V. Shank, Phys. Rev. Lett. 56, 761 (1986).

${ }^{38}$ F. A. Lindemann, Phys. Z. 11, 609 (1910).

${ }^{39}$ J. F. Vetelino, S. P. Gaur, and S. S. Mitra, Phys. Rev. B 5, 2360 (1972).

${ }^{40}$ CRC Handbook of Chemistry and Physics, edited by D. E. Lide, 87th ed. (CRC, Boca Raton, 2006-2007).

${ }^{41}$ J. Larsson, A. Allen, P. H. Bucksbaum, R. W. Falcone, A. Lindenberg, G. Naylor, T. Misalla, D. A. Reis, K. Scheidt, A. Sjögren, P. Sondhauss, M. Wulf, and J. S. Wark, Appl. Phys. A: Mater. Sci. Process. 75, 467 (2002).

${ }^{42}$ C. R. Wie, T. A. Tombrello, and T. Vreeland, Jr., J. Appl. Phys. 59, 3743 (1986).

${ }^{43}$ F. Rieutord, Acta Crystallogr., Sect. A: Found. Crystallogr. 46,
526 (1990).

${ }^{44}$ A. Rousse, C. Rischel, S. Fourmaux, I. Uschmann, S. Sebban, G. Grillon, Ph. Balcou, E. Förster, J. P. Geindre, P. Audebert, J. C. Gauthier, and D. Hulin, Nature (London) 410, 65 (2001).

${ }^{45}$ W. H. Press, S. A. Teukolsky, W. T. Vetterling, and B. P. Flannery, Numerical Recipes in $C++$ (Cambridge University Press, Cambridge, 2002).

${ }^{46}$ K. T. Tsen, J. G. Kiang, D. K. Ferry, and H. Morkoç, Appl. Phys. Lett. 89, 112111 (2006).

${ }^{47}$ M. A. Stroscio and M. Dutta, Phonons in Nanostructures (Cambridge University Press, Cambridge, 2001).

${ }^{48}$ A. Matulionis, Phys. Status Solidi A 203, 2313 (2006).

${ }^{49}$ A. D. Polyanin, Handbook of Linear Partial Differential Equations for Engineers and Scientists (Chapman and Hall, London/ CRC, Boca Raton, 2002).

${ }^{50}$ In the case of parabolic band approximation, $\Delta E_{e}=(\hbar \omega$ $\left.-E_{g}\right) /\left(1+m_{e} / m_{h}\right)$ and $\Delta E_{h}=-\left(m_{e} / m_{h}\right) \Delta E_{e}$, where $m_{e}$ and $m_{h}$ are the effective masses for electrons and holes.

${ }^{51}$ In the case of InSb, a laser pulse $(\hbar \omega \approx 1.55 \mathrm{eV})$ of fluence just below the melting threshold $\left(\sim 10 \mathrm{~mJ} \mathrm{~cm}^{-2}\right)$ creates an electron density on the order of $10^{21} \mathrm{~cm}^{-3}$. Because of the limited states in the $\Gamma$ valley $\left(\lesssim 10^{20} \mathrm{~cm}^{-3}\right)$, an intervalley scattering time $\tau_{\Gamma \rightarrow L}\left(\right.$ or $\tau_{\Gamma \rightarrow X}$ ) smaller than (or comparable to) the laser-pulse duration is needed to clear out the phase space for further transitions.

${ }^{52}$ Energy and momentum conservations impose constrains on the maximum wave vector $q_{\max }$ of the LO phonons that interact with the electrons. Conservation of energy states that $E_{c}\left(\mathbf{k}_{i}\right)-E_{c}\left(\mathbf{k}_{f}\right)$ $=\hbar \omega_{\mathrm{LO}}$ whereas conservation of momentum, $\mathbf{q}_{\max }=\mathbf{k}_{i}-\mathbf{k}_{f}$, with $\mathbf{k}_{i}$ and $\mathbf{k}_{f}$ standing for initial and final states of the electron in the $\mathbf{k}$ space. For InSb, with $E_{c}\left(\mathbf{k}_{i}\right) \approx 1.33 \mathrm{eV}$ and $\hbar \omega_{\mathrm{LO}}$ $\approx 0.024 \mathrm{eV}, q_{\max } \approx 3 \times 10^{7} \mathrm{~cm}^{-1}$.

${ }^{53}$ For parabolic bands $C_{e}$ can be calculated following chapter 2 in Ref. 21, with $H_{e}=F_{3 / 2}\left(\eta_{e}\right) / F_{1 / 2}\left(\eta_{e}\right)$, where $\eta_{e}=\left(\mu_{e}\right.$ $\left.-E_{\mathrm{CB}}\right) /\left(k_{B} T_{e}\right), \mu_{e}$ is the quasi-Fermi level for the electrons, and $F_{j}(\eta)$ is the Fermi-Dirac integral of the order $j$.

${ }^{54}$ Chapter 23, Eq. (23.29) of Ref. 21.

${ }^{55}$ For parabolic bands, $k_{B} T_{e} \sim 1 \mathrm{eV}$ and $N_{e}$ on the order of $10^{21} \mathrm{~cm}^{-3}, H_{e} \approx 1.2$

${ }^{56}$ The resulting differential equation for the displacement $u(z, t)$ is of the form $\partial^{2} u / \partial t^{2}=c_{1} \partial^{2} u / \partial z^{2}+g\left(c_{2}, z, t\right)$, where $g$ is an arbitrary function that does not depend on $u$, and $c_{1}$ and $c_{2}$ $\equiv\left\{c_{2}^{a}, c_{2}^{b}, \ldots\right\}$ are constants. An analytical solution of this type of equation can be found in Ref. 49.

${ }^{57}$ A possible surface damage has been ruled out by comparing the unperturbed rocking curves before and after laser excitation. Visible sample damage (very faint mark) was first observed at a laser fluence of about $11 \mathrm{~mJ} / \mathrm{cm}^{2}$ but there was no measurable loss in diffraction efficiency. The surface damage was considerable at about $14 \mathrm{~mJ} / \mathrm{cm}^{2}$ with about $10 \%$ unpumped diffraction loss over $30 \mathrm{~min}$.

${ }^{58}$ The optimal $\tau$ and its uncertainty $\Delta \tau$ are determined by minimizing the chi square with respect to $\tau$ and $\Delta \theta_{B}$ (Ref. 45). 